\title{
Optimization of a Piezoelectric Mechanical Amplifier Actuator for Nano-Indentation
}

\section{Pranati Purohit, Indu Elizabeth, S. Seela Kumar \& Sushil Kumar}

To cite this article: Pranati Purohit, Indu Elizabeth, S. Seela Kumar \& Sushil Kumar (2019) Optimization of a Piezoelectric Mechanical Amplifier Actuator for Nano-Indentation, Integrated Ferroelectrics, 202:1, 144-150, DOI: 10.1080/10584587.2019.1674832

To link to this article: https://doi.org/10.1080/10584587.2019.1674832

曲 Published online: 30 Dec 2019.

Submit your article to this journal $₫$

山 Article views: 20

Q View related articles $\widetilde{ }$

View Crossmark data 


\title{
Optimization of a Piezoelectric Mechanical Amplifier Actuator for Nano-Indentation
}

\author{
Pranati Purohit $^{\mathrm{a}}$, Indu Elizabeth ${ }^{\mathrm{b}}$, S. Seela Kumar ${ }^{\mathrm{b}}$, and Sushil Kumar ${ }^{\mathrm{a}, \mathrm{b}}$ \\ a Department of Applied Sciences, The NorthCap University, Gurugram, Haryana, India; ${ }^{\text {bCSIR-National }}$ \\ Physical Laboratory, New Delhi, India
}

\begin{abstract}
In the present investigation, a piezo-stack actuator coupled with a flexible diamond shaped frame has been investigated for its mechanical amplification property. The piezo-stack under electrical excitation produces a longitudinal strain along major diagonal of the frame which gets amplified due to its diamond shape along the shorter diagonal. The effect of the thickness of flexural hinges used in the diamond frame on amplification has been studied with a view to optimize the stress and displacement. The effectiveness of the piezoelectric mechanical amplifier actuator as a nano-indenter has been studied and discussed in this paper.
\end{abstract}

\section{ARTICLE HISTORY}

Received 1 October 2018

Accepted 16 September 2019

\section{KEYWORDS}

Piezo-force actuators; mechanical amplifier; flexure hinge nanoindentation

\section{Introduction}

Piezoelectric stacks can generate large displacements or forces [1], static or dynamic [2] which makes them ideal for driving precision-positioning stages [3] as for AFM, photolithography, robotic manipulators, auto-focusing of optical systems, etc. and for application of low precision forces [4] for nano-indentation characterization of thin films and many other applications. In order to have the speed and high bandwidth of piezo-positioning stacks high it necessitates to keep the dimensions of the stack small. However, compact stack-type actuators [5] typically generate displacements of the order of few microns without any blocking force. To generate force as well as higher displacements it becomes imperative to use mechanical displacement amplifiers. The technique of flexure hinge is used to amplify the displacement of piezoelectric actuators. Flexure hinges are advantageous as compared to pivotal hinges as there is no motion involved, hence no friction, no back lash, self-sealed i.e.free from dirt. As a result, flexure hinges are compatible with the high resolution and high-speed response of piezoelectric actuators.

Two types of flexure hinges are generally used and these are circular flexure hinges (Figure 1) and beam flexure (Figure 2) hinges. Circular hinges provide advantage in force application as they are stiffer as compared to beam flexure, thus fulfilling the requirement to generate force as well as displacement with amplification. Moreover, circular hinges are even stiffer in transverse direction thereby keeping the displacement free from any twists. In addition, their centers act as motionless pivots with minimum 


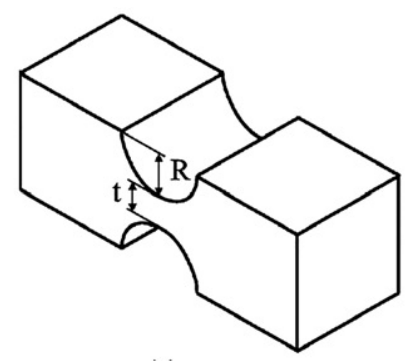

Figure 1. Right circular flexure.

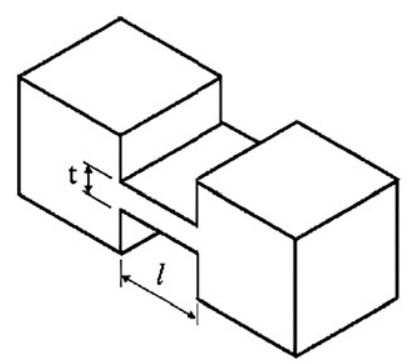

Figure 2. Beam flexure.

transverse motion of the point of deflection. For the design of mechanical amplifiers using flexure hinges it is required to know the dependence of the bending stiffness on the transverse-longitudinal ratio. From the literature it is difficult to describe the dependence of the bending compliance of hinges on its thickness as there several expressions available valid for different ranges of transverse-longitudinal ratio which yield results with various degrees of error from the values obtained by finite element analysis. Present study is an attempt to compare some relevant power laws of stiffnessthickness dependence and to explain some experimental results from the literature.

\section{Expression for Bending Compliance}

For the flexure hinges finite element analysis and corresponding empirical stress/strain relationships have been derived by many workers. Smith et al. [6] have derived the following equation to calculate the bending angular compliance and $y$ - deflection of right circular hinges, which has low error from FEA results and is valid over a wide range of values of transverse-longitudinal ratio.

$$
\frac{\alpha_{z}}{M_{z}}=\frac{(3.984 R+13.56 t)}{E b t^{3}}
$$

Over a broad range of values of $\frac{t}{R}, 0.2$ to 0.65 . Here $\alpha_{z}$ is the angular deflection and $M_{z}$ is the moment of the force applied at one end in the direction perpendicular to the plane of the hinge. The proportional error of $\frac{\alpha_{z}}{M_{z}}$ grows about $10 \%$ over the range. In another approximation by Paros and Bord [7], the expression for bending angular compliance is given as 


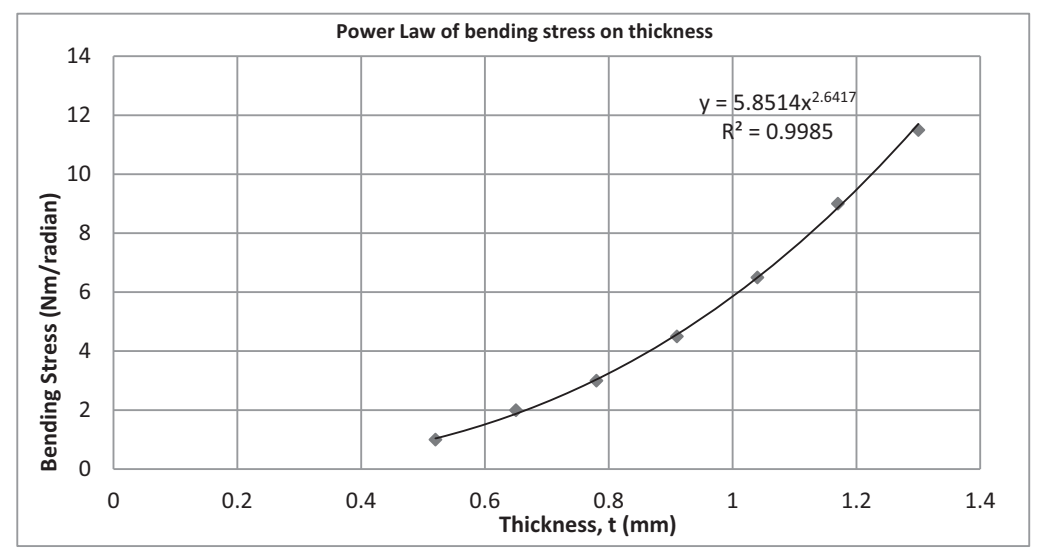

Figure 3. Power law of bending stress of a right circular flexure beam fdata taken from ning $\mathrm{Xu}$ et al. (2017)\}[8].

$$
\frac{\alpha_{z}}{M_{z}}=\frac{9 \pi R^{1 / 2}}{2 E b t^{5 / 2}}
$$

This approximation is valid over a small range of values of $\frac{t}{R}$ from 0.05 to 0.1 . In expressions (1) and (2) $E$ is the Young's modulus of the material of the hinge, $b$ is hinge width, $R$ is the radius of curvature, and $t$ is the thickness. Equations (1) and (2) clearly show that the dependence of the compliances of the hinge on its thickness has widely varying power exponent. On the other hand the bending compliance for a beam flexure hinge shows yet another dependence on power of $t$.

$$
\frac{\alpha_{z}}{M_{z}}=\frac{12 L}{E t^{3} b}
$$

The y- deflection $\Delta y$ per unit force applied $F_{y}$ for right circular hinge is given by

$$
\frac{\Delta y}{F_{y}}=R^{2} \frac{\alpha_{z}}{M_{z}}
$$

The equation (1) can be expanded to study the dependence of the bending stiffness and hence that of $y$ - deflection on the thickness of the hinge as follows.

$$
\frac{\alpha_{z}}{M_{z}}=\frac{3.984 R}{E b t^{3}}+\frac{13.56}{E b t^{2}}
$$

and

$$
\frac{\Delta y}{F_{y}}=\frac{3.984}{E b\left(\frac{t}{R}\right)^{3}}+\frac{13.56 t}{E b\left(\frac{t}{R}\right)^{3}}
$$

Clearly, two regions are to be distinguished viz. the first term of $t^{3}$ dependence representing in small thickness approximation the longitudinal deformation regime and the second term of $t^{2}$ dependence representing an increasing linear in thickness contribution higher stiffness due to shear deformation.

We can observe thickness behavior in the experimentally obtained bending stiffness \{data taken from Ning Xu et al (2017)\}[8] shown in Figure 3. It is seen that the data 


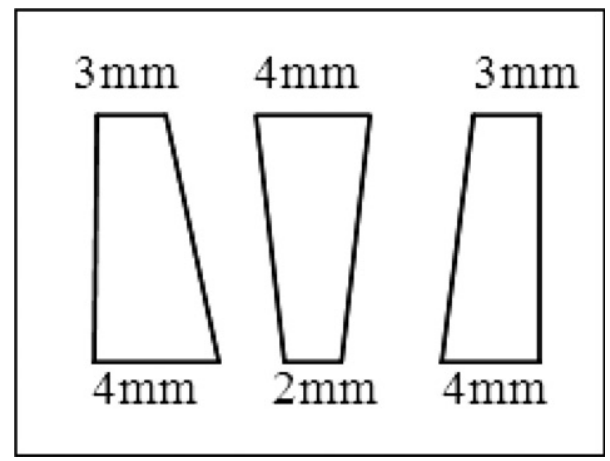

Figure 4. Tapered aluminum pieces used for generating pre stress in the piezo-actuator.

fits well with a power exponent of about 2.6, that is between the powers of 2 and three of the equation (1).

\section{Description of a Fabricated Piezo-Amplifier Actuator}

The design of an axial piezo-actuator using stacks of piezoelectric elements together with a diamond shaped mechanical amplifier [9]. The design uses piezoelectric elements along with metallic electrodes between elements so that when stacked together alternate pair of elements get connected in parallel. These stacks are pre-stressed in compression so as to ensure that the stack remains under compression over the complete range of operation of the actuator. The design of the amplifier is diamond shaped with sides in the form of cantilevers rather than hinges used in some other designs. These features give the piezoactuator a robust structure which can withstand extraneous stresses very well.

The piezo-actuator is based on 14 square plates (elements) of piezoelectric ceramic material equivalent to PZT-5 having $15 \mathrm{~mm}$ sides and $1 \mathrm{~mm}$ thickness. PZT-5 is considered because of its large $\mathrm{d}_{33}\left(400 \times 10^{-12} \mathrm{pC} / \mathrm{N}\right)$ piezoelectric and comparatively low cost.

Tapered aluminum washers of dimensions and shape as shown in the Figure 4 are machined for used to generate pre stress along the long dimension of the piezo-actuator. The washers when fully in place in the stack could generate a compression of the stack up to $2 \mathrm{~mm}$. The stack in the present case was assembled with the washers partially aligned so as to compress the stack outward longitudinal and the associated lateral inward displacement by about $0.2 \mathrm{~mm}$ so that the stack remains under compression for the complete span of actuation under electrical excitation by $\pm 0.1 \mathrm{~mm}$. A diamond shaped frame (as shown in Figure 5) having a long axis of $90 \mathrm{~mm}$ and short axis of $40 \mathrm{~mm}$ was designed and fabricated on a wire-cut machine. The piezoelectric stacks were mounted inside it along the long axis with the tapered aluminum pieces so that the stacks are brought into pre-compression. When an external voltage is applied to the stacks, they undergo either contraction or expansion depending on the polarity of the applied voltage.

\section{Working Principle}

The stacked piezo-element actuators generate moderate displacement with large blocking force. The displacement generated in a stack due to an applied voltage can be expressed as 


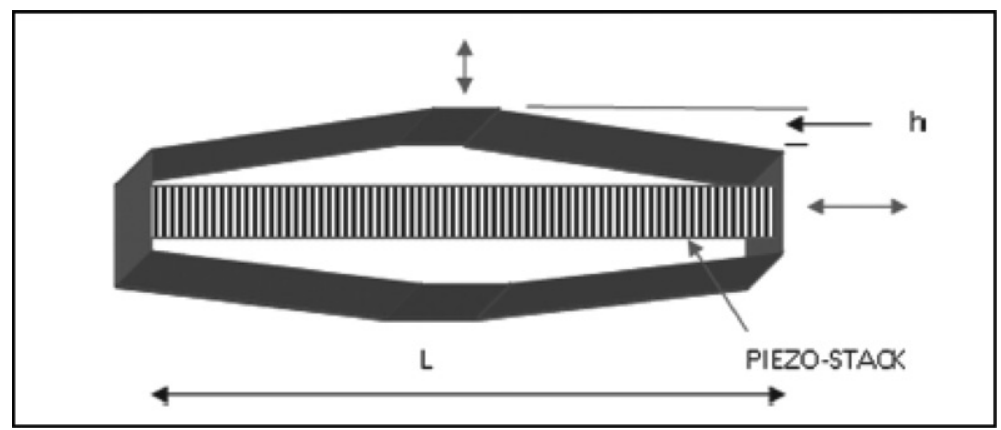

Figure 5. A schematic of the diamond shaped frame with long axis of $90 \mathrm{~mm}$ and short axis $40 \mathrm{~mm}$.

$$
\delta=n d_{33} V
$$

Where, $n$ is the number of piezoelectric elements in the stack, $d_{33}$ is the piezoelectric strain constant and $V$ is the applied voltage.

There are 14 elements in the stack. For an excitation voltage of $900 \mathrm{~V}$ each piezoelement would experience a longitudinal displacement of $0.27 \mu \mathrm{m}$ considering the $d_{33}$ value of PZT-4 material is approximately $300 \mathrm{pC} / \mathrm{N}$. elements the total longitudinal displacement of the stack corresponding to 45 elements would be $0.27 \times 14=3.8 \mu \mathrm{m}$.

The angle of the hinge is about 15 degree. The amplification factor for the lateral displacement for this angle would be $\tan ^{-1}(15)^{\circ}=3.9$ for each hinge. For two hinges in series, it would be half of it.

Total lateral displacement would be $=3.8 \times 3.9 / 2=7.4 \mu \mathrm{m}$.

The blocking force corresponding to this displacement of the cantilever can be calculated from the formula of equation (1) for hinge width of the order of the radius of circular flexure given as

$$
\frac{\Delta y}{F_{y}}=\frac{13.56}{E b\left(\frac{t}{R}\right)^{2}}
$$

$R$ is the radius of the hinge of the diamond shaped frame, $t$ is the thickness of the frame, $b$ is the width of the frame, $E$ is the Young's modulus of the diamond shaped steel frame and $F$ is the force.

Here $R=2 \mathrm{~mm}, t=1.5 \mathrm{~mm}, E=2 \rtimes 10^{11} \mathrm{~N} / \mathrm{m}^{2}$ and $b=15 \rtimes 10^{-3} \mathrm{~m}$

Corresponding to a displacement of $0.074 \mathrm{~mm}$ of the cantilever the force can be calculated using (8) as

$\mathrm{F}=8 \mathrm{~N}$

\section{Experimental Procedure}

The piezoelectric stacks together with the aluminum washers and the diamond shaped frame were assembled as shown in the Figure 6. The assembled actuator was fixed with one of the square holders in the middle of the cantilever in a vise. The displacement of the piezo-actuator at the other holder was measured using a micro-dial gauge of resolution $1 \mu \mathrm{m}$, fixed in a clamp stand. 


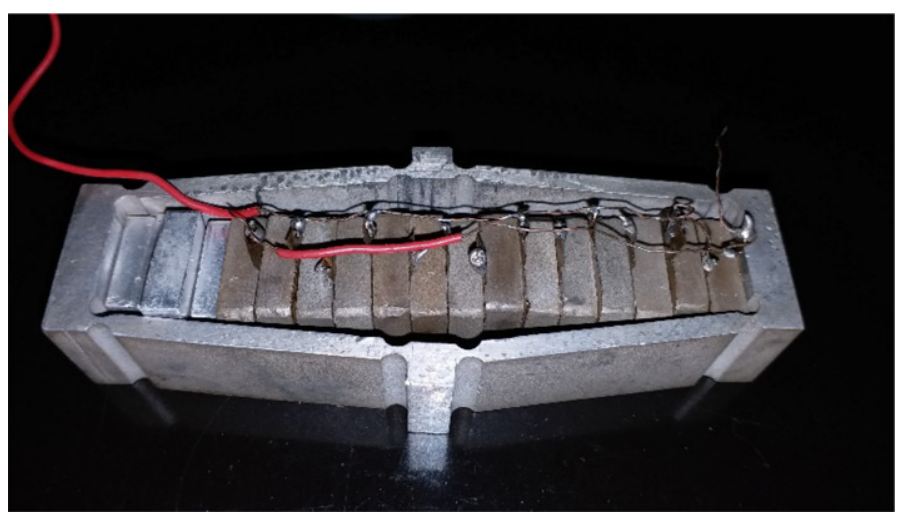

Figure 6. The designed diamond shaped piezo-electric actuator with stacks of piezoelectric elements and the aluminum pieces.

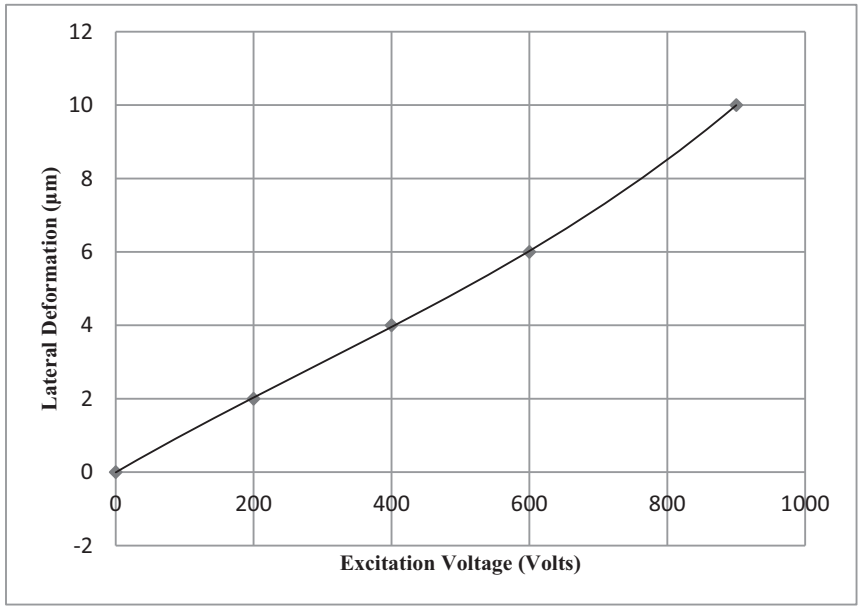

Figure 7. The plot of excitation voltage vs lateral deformation.

The displacement of the piezo-actuator subjected to a high voltage using a power supply was measured using a micro-dial gauge. The variation of lateral deformation of the actuator with excitation voltage is shown in Figure 7 which the authors have reported in a previous publication [4]. The performance of the actuator agrees with the computed displacement. Further, the displacement of a few microns along with a the blocking force of few newton can be achieved using the actuator which is seen sufficient for nano-indentation of thin films as reported by Huang et al. (2012) [10]. Piezo-actuators provide a means of applying force for precision measurement of nano-indentation with high resolution in a controlled repeatable manner.

\section{Concluding Remarks}

The performance of a designed and fabricated piezo-actuator amplifier using a diamond shaped frame having circular flexure hinges has been studied. The dependence of bending stiffness of right circular flexure hinge on its minimum thickness has been studied 
based on its expressions obtained by various workers. An effective expression corresponding to the experimental data from the literature has been discussed and used to design an actuator suitable for application in nano-indentation of materials.

\section{References}

1. G. H. Haertling, Rainbow ceramics-a new type of ultra-high displacement actuator, Am. Ceram. Soc. Bull. 73 (1), 93 (1994).

2. P. K. Panda et al., Electromechanical and dynamic characterization of in-house-fabricated amplified piezo actuator, Smart Mater. Res. 2012, 1 (2012). DOI: 10.1155/2012/203625.

3. M. Pozzi, and T. King, Piezoelectric actuators in micropositioning, Eng. Sci. Educ. J. 10 (1), 31 (2001). DOI: 10.1049/esej:20010105.

4. S. K. Jain, and S. S. K. Titus, Performance of a piezoelectric force actuator in low force measurement application, J. Mechatron. Mechatr. 1 (2), 109 (2013). DOI: 10.1166/jom.2013. 1018.

5. P. Purohit et al., Design and study of a piezoelectric actuator with mechanical amplification, 2nd National Conference on Recent Developments in Electronics (NCRDE-2016) University of Delhi, South Campus, 17-18 Feb, 2017, pp. 30-33.

6. S. Smith, D. Chetwynd, and D. Bowen, Design and assessment of monolithic high precision translation mechanisms, J. Phys. E: Sci. Instrum. 20 (8), 977 (1987). DOI: 10.1088/00223735/20/8/005.

7. J. Paros, and L. Weisbord, How to design flexure hinge, Mach Design 37, 151 (1965).

8. X. Ning, D. Ming, and Z. Xiaoqin, Analysis and design of symmetric notch flexure hinges, Adv. Mech. Eng. 9 (11), 1 (2017). DOI: 10.1177/1687814017734513.

9. Z. Huixing, and H. Brian, Analysis of a diamond shaped mechanical amplifier for a piezo actuator, Int. J. Adv. Manufact. Technol. 32 (1-2), 1 (2007).

10. H. Hu et al., Design and analysis of the precision-driven unit for nano-indentation and scratch test, J. Manufact. Syst. 31 (1), 76 (2012). DOI: 10.1016/j.jmsy.2011.01.005. 